\title{
Minimum superficial gas velocity for onset of foaming
}

\author{
Laurent Pilon $^{\mathrm{a}, *}$, Raymond Viskanta ${ }^{\mathrm{b}}$ \\ ${ }^{a}$ Mechanical and Aerospace Engineering Department, University of California, Los Angeles, CA 90095, USA \\ ${ }^{\mathrm{b}}$ School of Mechanical Engineering, Purdue University, West Lafayette, IN 47907, USA
}

Received 31 May 2002; received in revised form 20 January 2003; accepted 20 January 2003

\begin{abstract}
This paper is concerned with semi-batch foams generated by injecting gas bubbles in a vertical column containing a liquid phase at rest. Its aim is to better understand the physical mechanisms responsible for foam formation at the liquid free surface and to predict the superficial gas velocity for onset of foaming. The model for predicting the onset of foaming is derived from the one-dimensional drift-flux model for gravity driven flow in the absence of wall shear. The analysis is based on experimental data reported in the literature and covers a wide range of physico-chemical properties, bubble sizes and shapes, and flow regimes. It identifies the inhibition of coalescence between rising bubbles and bubbles at rest at the free surface as a key mechanism responsible for the onset of foaming. A semi-empirical correlation for high viscosity fluids has been developed and good agreement with experimental data is found.
\end{abstract}

(C) 2003 Elsevier B.V. All rights reserved.

Keywords: Foam separation; Materials processing; Bioreactors; Foam fractionation; Slag foaming; Glass foam

\section{Introduction}

Semi-batch foams or pneumatic foams are produced by injecting a continuous stream of gas bubbles at the bottom of a vertical column containing a foaming liquid at rest. Such foams are encountered in a number of practical technological systems ranging from chemical and materials processing, to bioreactors and separation of solid or liquid solutes from a solvent. Bubbles are either generated by chemical reactions taking place within the liquid or injected in the liquid. Foam can be desirable such as in bioreactors where it acts as a cushion preventing bursting bubbles from damaging the cells at the liquid surface or in electric arc furnaces where it is often required to shield the refractories from the arc, to protect the liquid metal from the atmosphere [1], and to help to stabilize the arc in modern electric arc furnaces [1]. In protein separation, proteins acting as surfactants concentrate in the foam that is collected to produce a solution with higher protein concentration [2]. In food processes or in glass melting furnaces, foam is undesirable since it may disrupt the production and significantly

\footnotetext{
* Corresponding author. Tel.: +1-310-206-5598; fax: +1-310-206-4830

E-mail address: pilon@seas.ucla.edu (L. Pilon).
}

affect the product quality and the energy efficiency of the process [3].

In one-dimensional two-phase flow, the area-averaged superficial velocities for the gas and liquid phases denoted as $j_{f}$ and $j_{g}$, respectively, can be expressed as a function of the area-averaged velocities of the liquid $v_{f}$ and gas phase $v_{g}$ and of the area-averaged void fraction $\alpha$ [4]:

$j_{g}=\alpha v_{g} \quad$ and $\quad j_{f}=(1-\alpha) v_{f}$

The superficial velocities of the gas and the liquid phases can also be expressed as a function of the volumetric flow rates:

$j_{g}=\frac{Q_{g}}{A} \quad$ and $\quad j_{f}=\frac{Q_{f}}{A}$

where $Q_{g}$ and $Q_{f}$ are the volumetric flow rates of the gas and liquid phases, respectively, while $A$ is the cross-sectional area of the container. Thus, the superficial velocities $j_{g}$ and $j_{f}$ can be easily monitored experimentally. Finally, the velocity of the center of volume of the mixture $j$ can be expressed as

$j=j_{g}+j_{f}$

The so-called drift velocity is defined as the relative velocity of the gas phase with respect to the velocity of the center of volume of the mixture [4]. The drift velocity $V_{g j}$ 
and the associated drift-flux $j_{g f}$ are given, respectively, by $[4,5]$ :

$V_{g j} \equiv v_{g}-j \quad$ and $\quad j_{g f} \equiv \alpha V_{g j}$

The drift-flux $j_{g f}$ for different two-phase flow regimes can be written in the general form as [4]:

$j_{g f}=K v_{\infty} \alpha(1-\alpha)^{n}$

where $K$ is a parameter depending on the average bubble radius and $v_{\infty}$ is the velocity of a single bubble of radius $r$ rising in a quiescent liquid. The present study is concerned with a gas-liquid flow with the liquid phase at rest. Thus, the superficial liquid velocity vanishes, i.e. $j_{f}=0$. Then, according to Eq. (3) the area-averaged superficial gas velocity $j_{g}$ equals $j$. Then, from Eqs. (3) and (4) the drift-flux can be expressed as:

$j_{g f}=(1-\alpha) j_{g}$

Fig. 1 shows the drift-flux $j_{g f}$ plotted versus the void fraction $\alpha$ as given by Eq. (5) (solid line) and Eq. (6) (dashed line). One can observe that for any positive superficial gas velocity $j_{g}$, Eqs. (5) and (6) are simultaneously satisfied for two different values of the void fraction, i.e. there are two functioning points as previously discussed by Wallis [4]. The first functioning point falls into the bubbly flow regime (low void fraction) and the second functioning point into the foamy regime (large void fraction). The foam occupies the upper part of the column and coexists with a bubbly region at the lower part as observed experimentally.

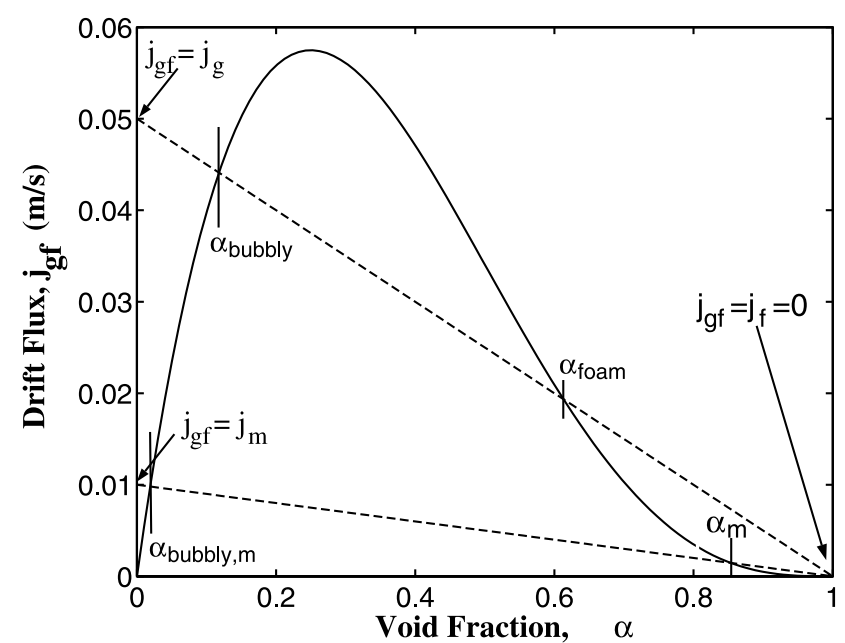

Fig. 1. Functioning points obtained by Eq. (5) (solid line) and Eq. (6) (dashed line) for superficial gas velocities $j_{g}$ of $0.01 \mathrm{~m} / \mathrm{s}$ and $0.05 \mathrm{~cm} / \mathrm{s}$ with $j_{f}=0 \mathrm{~m} / \mathrm{s}$.

Experimental observations also indicate that pneumatic foams do not form for any arbitrarily small gas flow rate. Laimbock [6] has observed that a minimum superficial gas velocity should be reached to initiate foaming of molten glass. The same observations have been made for different aqueous solutions [7-12], as well as for molten steel [1,13-18]. Fig. 2 shows a typical plot of the steady-state foam thickness $H_{\infty}$ as a function of the superficial gas

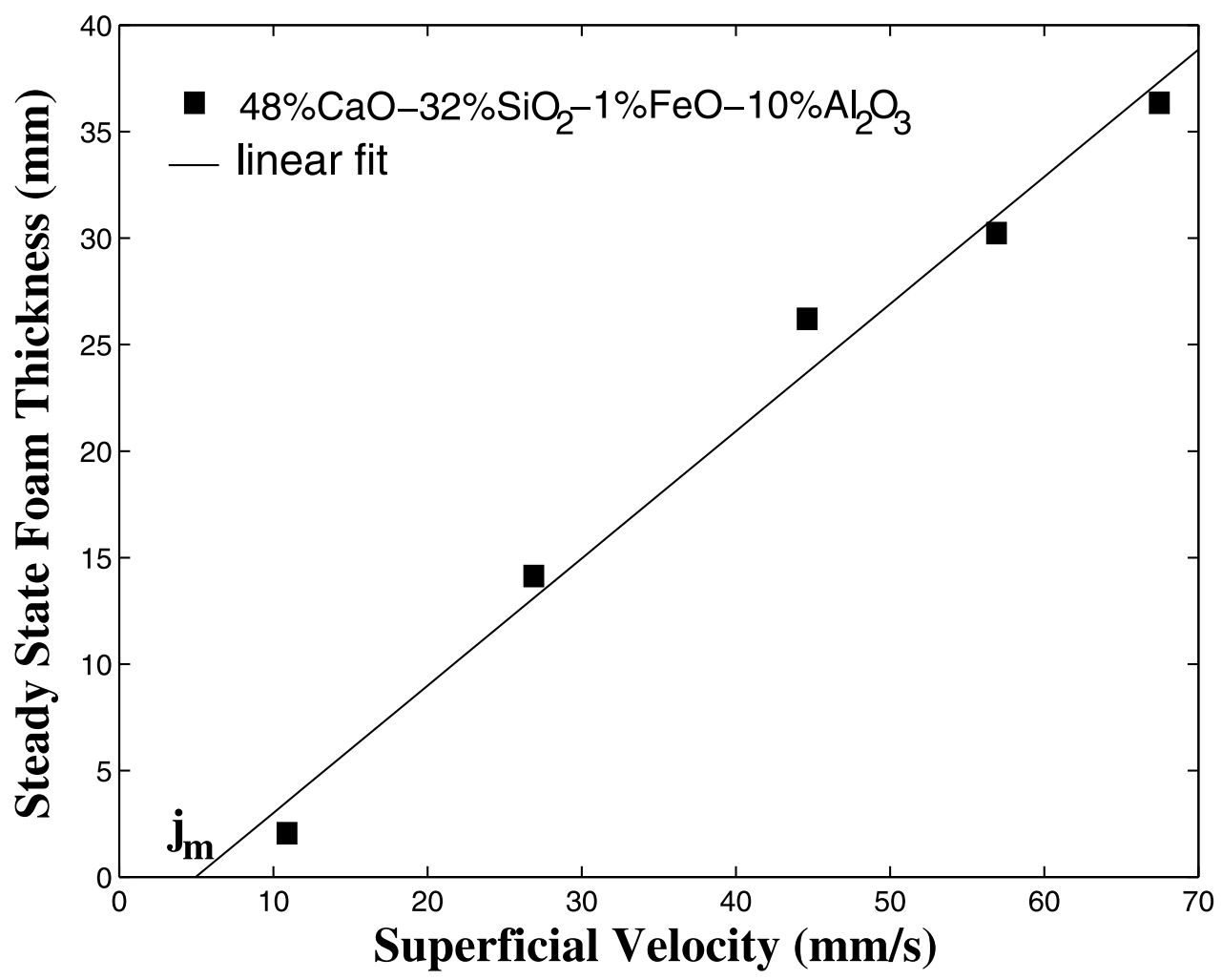

Fig. 2. Typical plot of the steady state foam thickness vs. superficial argon velocity [18]. 
velocity $j_{g}$ and demonstrate the existence of a minimum superficial gas velocity for onset of foaming $j_{m}$. However, the drift-flux model predicts that the two functioning points always exist. Hence, a foam layer should form for any arbitrary small superficial gas velocity. This conclusion is obviously in contradiction with experimental observations discussed previously. Wallis attributed this to the instability of the foam leading to 'rapid bubble bursting and agglomeration' [4]. The coexistence of a slug flow and a foam layer at the top of the bubble column has not been observed experimentally due to both reduced number of small bubbles and the agitation caused by slug bubbles bursting at the free surface and breaking the foam layer.

Whether foam is desirable or not, it is of fundamental and practical interest to understand the foaming process and to predict the conditions under which foam starts forming in order to operate a process under the most favorable conditions. The minimum superficial gas velocity for onset of foaming $j_{m}$ should be determined as a function of the physico-chemical properties of the two phases and the operating conditions. However, to the best of our knowledge, no self-contained model able to predict the minimum superficial gas velocity for onset of foaming $j_{m}$ has been reported in the literature. The only attempt has been to determine the transition from the homogeneous bubbling regime to the foaming regime on a flow map plotting the void fraction versus the Froude number [12]. The map predicts 'an estimate' of the minimum superficial gas velocity for onset of foaming as a function of the void fraction in the bubbly flow and of the container diameter. Moreover, the drift-flux model alone fails to explain the existence of a minimal superficial gas velocity for onset of foaming as observed experimentally.

The objective of the present work is to provide physical explanations of the experimental facts and develop a quantitative self-contained model which gives the minimum superficial gas velocity as a function of the physico-chemical properties of the two phases and the operating conditions by using the drift-flux model and paying particular attention to bubble coalescence.

\section{Analysis}

\subsection{Physical phenomena}

In gas-liquid flow, bubbles of different sizes and velocities may collide resulting in the thinning of the film separating them. Collisions between two bubbles may lead either to the coalescence due to the rupture of the film or to bouncing and separation of the bubbles [19]. The coalescence rate of bubbles depends on the frequency of collision and on the probability that bubbles coalesce upon collision. The frequency of collisions depends on the liquid flow and on the hydrodynamic interactions between the bubbles and the liquid phase [20]. On the other hand, coalescence upon collision takes place when the collision duration time $t_{c}$ is larger than the time to drain the film between bubbles $t_{d}$. In the limiting cases, the thinning of the film separating two colliding bubbles is dominated by either viscous or inertial forces. Finally, the probability of coalescence $P$ should tend to unity when the ratio $t_{d} / t_{c}$ is small and to zero when the ratio $t_{d} / t_{c}$ is large. An expression for the probability of coalescence as a function of the collision duration time $t_{c}$ and the drainage time $t_{d}$ has been suggested [20]:

$P=\exp \left(-t_{d} / t_{c}\right)$

The Weber number is commonly used in the studies of bubble coalescence $[11,19,21]$ and represents the ratio of the inertial forces to the surface tension forces [19]:

$W e=\frac{\rho_{f} V^{2} r}{\sigma}$

where $V$ is the relative velocity of centers of colliding bubbles. In pure water, Duineveld [19] observed three different bubble interaction behaviors: (1) when the Weber number based on the relative approaching velocity and denoted $W e_{a}$ is less than 0.18 , bubbles coalesce; (2) for $W e_{a}$ larger than 0.18 , and for Weber number $W e_{\infty}$ based on the terminal velocity of a single bubble $v_{\infty}$ less than 3.3, bubbles bounce at the first contact but eventually coalesce; (3) for $W e_{a}$ and $W e_{\infty}$ larger than 0.18 and 3.3, respectively, bubbles bounce at the first collision and separate. Finally, experimental observations in low viscosity liquids show that bubble coalescence is inhibited when surfactants or electrolytes are added to the pure solution and is even terminated above a certain surfactant concentration [19,21,22].

Similarly, a single bubble reaching a free interface can either merge with the interface almost instantaneously (for small approaching velocities) or bounce back one or several times before stabilizing at the free interface to finally burst (for large approaching velocities). Kirkpatrick and Lockett [21] found that for Weber numbers larger than 0.5 (based on the bubble velocity) bubbles bounce one or several times before coalescing with the interface. They also showed that bubble coalescence is identical in doubly distilled water and in tap water. However, the presence of electrolyte inhibited coalescence of the bubble with the free interface [21].

Chesters [20] proposed an expression for the collision duration time $t_{c}$ and for the drainage time $t_{d}$ in both the viscous and the inertial dominated limiting cases assuming that bubbles have the same radius and both gas viscosity and van der Waals forces can be ignored. In each limiting case, the ratio of the collision duration time $t_{c}$ and to drainage time $t_{d}$ can be written as [20]:

$$
t_{d} / t_{c}=\left(\frac{\rho_{f} V^{2} r}{32 \sigma}\right)^{1 / 2}
$$

for inertia controlled drainage $\left(\operatorname{Re}_{\infty} \leq 24\right)$, and 


$$
t_{d} / t_{c}=\frac{3 \mu}{\sqrt{2 \sigma \rho_{f} r}}
$$

for viscosity controlled drainage $\left(R e_{\infty}>24\right)$

where $\sigma$ is the surface tension of the gas-liquid system and $\mu_{f}$ and $\rho_{f}$ are the dynamic viscosity and the density of the liquid phase, respectively. The Reynolds number of a single bubble of radius $r$ rising in an infinite liquid with the terminal velocity $v_{\infty}$ can be expressed as [23]:

$R e_{\infty}=\frac{2 r \rho_{f} v_{\infty}}{\mu_{f}}$

Finally $V$ is the relative velocity of centers of colliding bubbles and $r$ the average bubble radius. In the present work, $r$ is assumed to be the average bubble radius at the liquid free surface, thus accounting for eventual bubble growth due to pressure change and coalescence taking place between the injection system and the liquid surface. Then, $V$ is taken as the terminal velocity $v_{\infty}$ of a single bubble of average size rising in an infinite medium as suggested by Duineveld [19]. It can be interpreted as the relative velocity between the center of a rising bubble and that of a bubble at rest at the liquid free surface. The transition between the inertia and the viscosity controlled drainage regimes was assumed to occur when the corresponding characteristic time ratios given by Eqs. (9) and (10) are equal, i.e. when $R e_{\infty}=24$. The ratio of the collision duration time $t_{c}$ to the drainage time $t_{d}$ can be seen as the scaling of the inertial or the viscous forces to the surface tension forces. Note that for inertia dominated drainage, the ratio of the characteristic times $t_{d} / t_{c}$ is proportional to the square root of the dimensionless Weber number given by Eq. (8). It is also interesting to note that in the viscosity dominated regime, the ratio $t_{d} / t_{c}$ does not depend on the bubble velocity.

Finally, Fig. 1 indicates that the void fraction for the functioning point in the foamy regime decreases as the superficial gas velocity increases. This suggests that as the superficial gas velocity increases, the bubbles at the top of the liquid column are less packed and their frequency of collision is reduced. Moreover, as the superficial gas velocity increases, the average bubble velocity increases while the probability of coalescence upon collision decreases in the case of inertia dominated drainage. For viscosity controlled drainage, the probability of coalescence is independent of the superficial gas velocity. In summary, increasing the superficial gas velocity, reduces one or both components of the coalescence rate: (1) the frequency of collision and (2) the probability of coalescence for inertia dominated drainage. For a given combination of superficial gas velocity and void fraction, the coalescence rate is reduced enough to allow foam formation. Therefore, there exists a minimum superficial gas velocity and a corresponding maximum void fraction $\alpha_{m}$ for onset of foaming beyond which the foam is unstable and disappears instantaneously from the liquid interface. For void fraction smaller than $\alpha_{m}$, bubbles are stable and do not coalesce enabling the formation of a foam layer. The conditions for on- set of foaming are reached by increasing the superficial gas velocity beyond $j_{m}$, i.e. by reducing the void fraction above the liquid surface below $\alpha_{m}$.

\subsection{Modeling}

The drift-flux $j_{g f}$ has been identified as a key variable in dealing with sedimentation, fluidization, and extraction experiments [23] and is expressed, in general, in terms of the terminal velocity $v_{\infty}$ of a single bubble of radius $r$ rising in an infinite volume of liquid, of the area-averaged void fraction $\alpha$ of the two-phase mixture, and of the physico-chemical properties of the two phases [5].

As previously discussed, the present study is concerned with a gas-liquid flow for which the superficial liquid velocity vanishes, i.e. $j_{f}=0$. Moreover, the wall shear stresses are small and can safely be neglected as suggested by Wallis [4] and by Guitian and Joseph [24]. Therefore, the flow can be described as a vertical gravity dominated flow with no wall shear. Thus, the velocity and void fraction profiles at any given cross-section perpendicular to the two-phase flow are assumed to be uniform [4].

Expressions of the drift-flux $j_{g f}$ for different two-phase flow regimes along with the transition criteria as a function of the physico-chemical properties of the system can be found in the literature. The analysis by Ishii and co-workers $[23,25]$ is based on the dimensionless bubble radius $r^{*}$ and on the viscosity number $N_{\mu}$ which represents the scaling of the viscous forces by the surface tension forces:

$$
\begin{aligned}
& r *=r\left[\frac{\rho_{f}\left(\rho_{f}-\rho_{g}\right) g}{\mu_{f}^{2}}\right]^{1 / 3} \text { and } \\
& N_{\mu}=\frac{\mu_{f}}{\sigma}\left[\frac{\left(\rho_{f}-\rho_{g}\right) g \sigma}{\rho_{f}^{2}}\right]^{1 / 4}
\end{aligned}
$$

Three different regimes of gas-liquid systems are considered in the present study: (1) the viscous regime in which bubbles are spherical and a complete similarity exists between the expressions of the drag coefficient for a single particle system and for a multiparticle system, (2) the distorted particle regime in which the bubbles are distorted and the drag on an individual bubble is strongly affected by the wake generated by the other bubbles, and (3) the churn-turbulent regime in which the distorted bubbles influence not only the other bubbles but also the surrounding liquid. Expressions for the drift-flux $j_{g f}$ and for the terminal velocity $v_{\infty}$ are available for each one of these regimes [23].

The viscous regime, can be divided in two sub-regimes namely, the Stokes' flow regime and the wake regime. In the Stokes' flow sub-regime $\left(R e_{\infty} \ll 1\right)$ the drift-flux can be expressed as [23]:

$j_{g f}=v_{\infty} \alpha(1-\alpha)^{3}$ 
with

$v_{\infty}=\frac{2}{9} \frac{\left(\rho_{f}-\rho_{g}\right) g r^{2}}{\mu_{f}}$

In the wake sub-regime, i.e. for larger Reynolds numbers $\left(R e_{\infty} \geq 1\right)$, the drift-flux is given by [23]:

$j_{g f}=v_{\infty} \alpha(1-\alpha)^{3}\left[\frac{1+\psi\left(r^{*}\right)}{1+\psi\left(r^{*}\right)(1-\alpha)^{9 / 7}}\right]$

where $\psi\left(r^{*}\right)$ is a function of the dimensionless radius $r^{*}$ and is defined as:

$\psi\left(r^{*}\right)=0.55\left[\left(1+0.08 r^{* 3}\right)^{4 / 7}-1\right]^{0.75}$

The terminal velocity of a single bubble rising in an infinite medium $v_{\infty}$ is obtained from the force balance equating the buoyancy force to the drag force. The correlation for the drag coefficient as a function of the Reynolds number $R e_{\infty}$ proposed by Ishii and Zuber [23] is used and the following non-linear equation is solved for $v_{\infty}$ :

$\frac{8\left(\rho_{f}-\rho_{g}\right) g r}{3 \rho_{f} v_{\infty}^{2}}=\frac{24}{R e_{\infty}}\left(1+0.1 R e_{\infty}^{0.75}\right)$

Note that the Stokes' flow sub-regime is asymptotic to the wake regime in the limiting case when the Reynolds number $R e_{\infty}$ is much smaller than unity. The transition between the viscous regime and the distorted bubble regime is expressed in terms of the viscosity number at the transition denoted $\left.N_{\mu}\right|_{t}$ and given by:

$\left.N_{\mu}\right|_{t}=0.11\left[\frac{1+\psi\left(r^{*}\right)}{\psi\left(r^{*}\right)^{8 / 3}}\right]$

For $N_{\mu} \leq\left. N_{\mu}\right|_{t}$ the bubbles are spherical and the flow is in the viscous regime whereas for $N_{\mu}>\left.N_{\mu}\right|_{t}$ the bubbles are distorted and the flow is in the distorted bubble regime.

In the distorted bubble regime the drift-flux and the terminal velocity of a single bubble are expressed, respectively, as [23]:

$$
\begin{aligned}
j_{g f} & =v_{\infty} \alpha(1-\alpha)^{3}\left[\frac{18.67}{1+17.67(1-\alpha)^{9 / 7}}\right] \\
& \approx v_{\infty} \alpha(1-\alpha)^{1.75}
\end{aligned}
$$

and

$v_{\infty}=\sqrt{2}\left[\frac{\left(\rho_{f}-\rho_{g}\right) g \sigma}{\rho_{f}^{2}}\right]^{1 / 4}$

In the churn-turbulent regime, the drift-flux and the terminal velocity of a single bubble are given, respectively, by [23]:

$j_{g f}=v_{\infty} \alpha(1-\alpha)^{1 / 4}$

$v_{\infty}=\sqrt{2}\left[\frac{\left(\rho_{f}-\rho_{g}\right) g r}{\rho_{f}^{2}}\right]^{1 / 4}$

According to Ishii and Zuber [23], the transition from the distorted bubble regime to the churn-turbulent flow regime occurs for void fraction of 0.3 . However, they recognize that in case of batch processes, such as these presently under consideration, 'detailed coalescence mechanisms and surface contaminations become important in determining the transition criterion', and the distorted bubble regime can remain even at high void fractions like in foams.

A general expression of the drift-flux for all the above described two-phase flow regimes can be derived from Eqs. (13)-(22):

$j_{g f}=v_{\infty} f(r *) \alpha(1-\alpha)^{n}$

where $f\left(r^{*}\right)$ is a function of the dimensionless radius $r^{*}$ and of the flow regime. Table 1 summarizes the expression for the terminal velocity $v_{\infty}$ of a single bubble, the function $f\left(r^{*}\right)$, and the parameter $n$ for gas-liquid mixtures in the three different flow regimes considered.

As discussed by Wallis [4] and Ishii and Zuber [23], there exists a maximum value of the void fraction up to which expressions for the drift-flux $j_{g f}$ and hence Eqs. (13)-(22) are valid. This maximum value depends on the shape of the particles and the nature of their interactions [4]. For gas-liquid systems, the flexibility of the bubble/liquid interface leads to negligible particle-particle interaction forces and the above expressions for the drift-flux and for the superficial gas velocity for onset of foaming $j_{m}$ in the different flow regimes are still valid for values of the void frac-

Table 1

\begin{tabular}{|c|c|c|c|c|}
\hline Regime & Terminal velocity $\left(v_{\infty}\right)$ & $f(r)$ & Parameter $(n)$ & Transition criteria \\
\hline \multirow{3}{*}{ Viscous } & $\underline{2} \underline{\left(\rho_{f}-\rho_{g}\right) g r^{2}}$ & \multirow{2}{*}{1} & \multirow[t]{2}{*}{3} & \multirow{2}{*}{$N_{\mu} \leq\left. N_{\mu}\right|_{t}$ and $R e_{\infty} \ll 1$} \\
\hline & $\mu_{f}$ & & & \\
\hline & $\frac{8 \Delta \rho}{3 \rho_{f} v_{\infty}^{2}}=\frac{24}{\operatorname{Re}_{\infty}}\left(1+0.1 R e_{\infty}^{0.75}\right)$ & {$\left[\frac{1+\psi(r *)}{1+\psi(r *)(1-\alpha)^{9 / 7}}\right.$} & 3 & $N_{\mu} \leq\left. N_{\mu}\right|_{t}$ and $R e_{\infty}>1$ \\
\hline Distorted bubble & $\sqrt{2}\left[\frac{\left(\rho_{f}-\rho_{g}\right) g \sigma}{\rho_{f}^{2}}\right]^{1 / 4}$ & 1 & 1.75 & $N_{\mu}>\left.N_{\mu}\right|_{t}$ \\
\hline Churn-turbulent & $\sqrt{2}\left[\frac{\left(\rho_{f}-\rho_{g}\right) g \sigma}{\rho_{f}^{2}}\right]^{1 / 4}$ & 1 & $1 / 4$ & $r<\left[\sigma /\left(\rho_{f}-\rho_{g}\right) g\right]^{1 / 3}$ \\
\hline
\end{tabular}

Parameters for the prediction of the superficial gas velocity for onset of foaming as given by Eq. (24) 


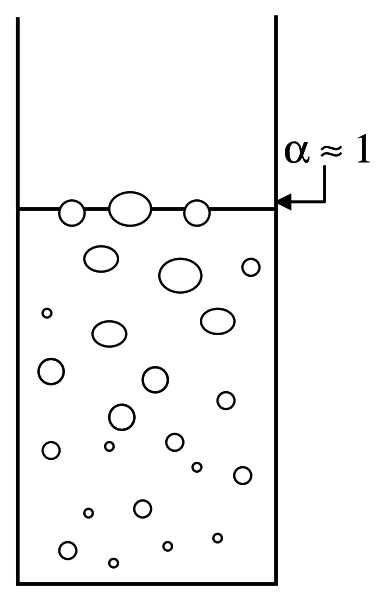

(a) $\mathrm{j}_{\mathrm{g}}<\mathrm{j}_{\mathrm{m}}$

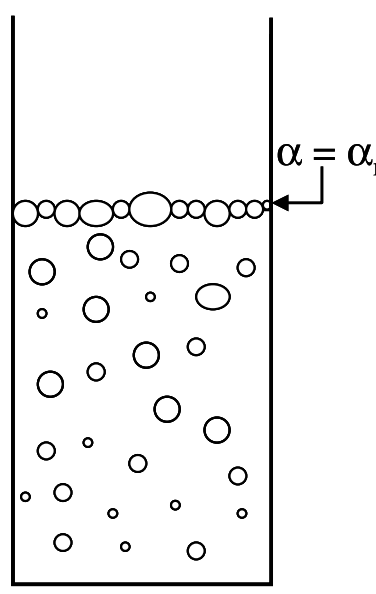

(b) $\mathrm{j}_{\mathrm{g}}=\mathrm{j}_{\mathrm{m}}$

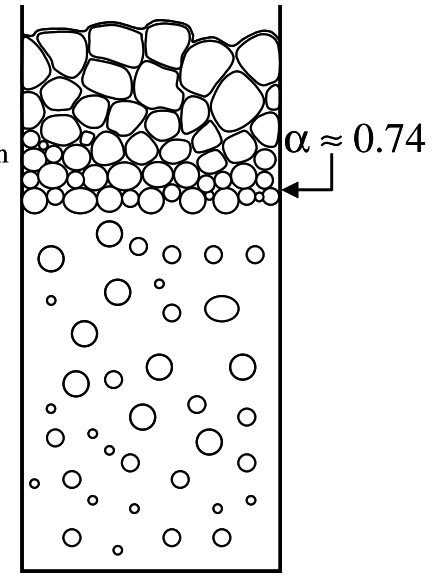

(c) $\mathrm{j}_{\mathrm{g}}>\mathrm{j}_{\mathrm{m}}$

Fig. 3. Schematic of the behavior of a foaming solution as the superficial gas velocity is increased (a) bubbly flow without foam, (b) onset of foaming, (c) developed foam layer.

tion $\alpha$ very close to unity, including foams or dense packing regimes $[4,23,25]$. Moreover, the drift-flux model has been validated for gas-liquid bubbly flow featuring void fraction close to unity such as foams $[4,11,23-26]$. Therefore, the drift-flux model can be used with confidence for predicting the onset of foaming since ideally, the onset of foaming corresponds to the situation when bubbly or churn-turbulent flow regimes with low void fraction prevail and coexist with only a single layer of packed bubbles accumulating at the free surface as shown in Fig. 3. Here $\alpha_{m}$ corresponds to the maximum void fraction for onset of foaming. Then, combining Eqs. (6) and (23) at the onset of foaming, i.e. when $\alpha=\alpha_{m}$, leads to the following expression for the superficial gas velocity for onset of foaming $j_{m}$ :

$j_{m}=v_{\infty} f\left(r^{*}\right) \alpha_{m}\left(1-\alpha_{m}\right)^{n-1}$

where $f\left(r^{*}\right), r^{*}$ are summarized in Table 1 .

Based on physical arguments, coalescence of rising bubbles with bubbles at rest at the liquid free surface has been identified as the main physical phenomena controlling foam formation. On the other hand, an expression for the minimum superficial gas velocity for onset of foaming $j_{m}$ has been derived from the drift-flux model as a function of the maximum void fraction for onset of foaming $\alpha_{m}$, operating conditions, and physico-chemical properties of the two phases. To confirm this analysis, the model predictions should be compared against experimental data.

\section{Results and discussions}

\subsection{Experimental data}

The experimental data collected by Pilon et al. [3] were used in the present study. Additional data were obtained from the literature [11]. The minimum superficial gas veloc- ity for onset of foaming $j_{m}$ was determined experimentally by extrapolating the data for the steady-state foam thickness $H_{\infty}$ as a function of the superficial gas velocity $j_{g}$ and by assuming a linear relationship [i.e. $H_{\infty} \propto\left(j_{g}-j_{m}\right)$ ] as suggested by Pilon et al. [3]. Fig. 2 shows a typical example of such an extrapolation used to determine $j_{m}$. The bubble shape and velocity $v_{\infty}$ as well as the associated value of the parameter $n$ were determined based on information summarized in Table 1. Note that the shape of the bubbles derived from Ishii's criteria agree with different models based on other dimensionless numbers than the viscosity number (see [27], p. 27). Even though no transition criteria between the distorted bubble and the churn-turbulent regimes has been given, experimental data reported by Pino et al. [11] were assumed to feature a churn-turbulent regime as described by the authors. The radii of the bubbles for data reported by these researchers were found based on the following correlation relating the bubble radius to the gas flow rate [see [4], Eq. (9.5), p. 245]:

$r=0.648\left(\frac{Q_{g}^{2}}{g}\right)^{1 / 5}$

In molten steel slags, the bubbles were assumed to reach their terminal velocity before reaching the free interface, i.e. in less than $50 \mathrm{~mm}$. This should be considered as a first order approximation that can be justified by the small values of the bubble sizes and terminal velocities. Finally, Eq. (24) indicates that the knowledge of either $j_{m}$ or $\alpha_{m}$ leads to the determination of the other. Finding $\alpha_{m}$ from $j_{m}$ appears to be more reliable in the present analysis since a small uncertainty in $\alpha_{m}$ can lead to a significant error in the value of $j_{m}$, particularly in the foamy regime. In contrast, uncertainty in the experimentally determined value of $j_{m}$ leads to a small variation in $\alpha_{m}$. Consequently, the maximum void fraction for onset of foaming $\alpha_{m}$ was obtained from the 


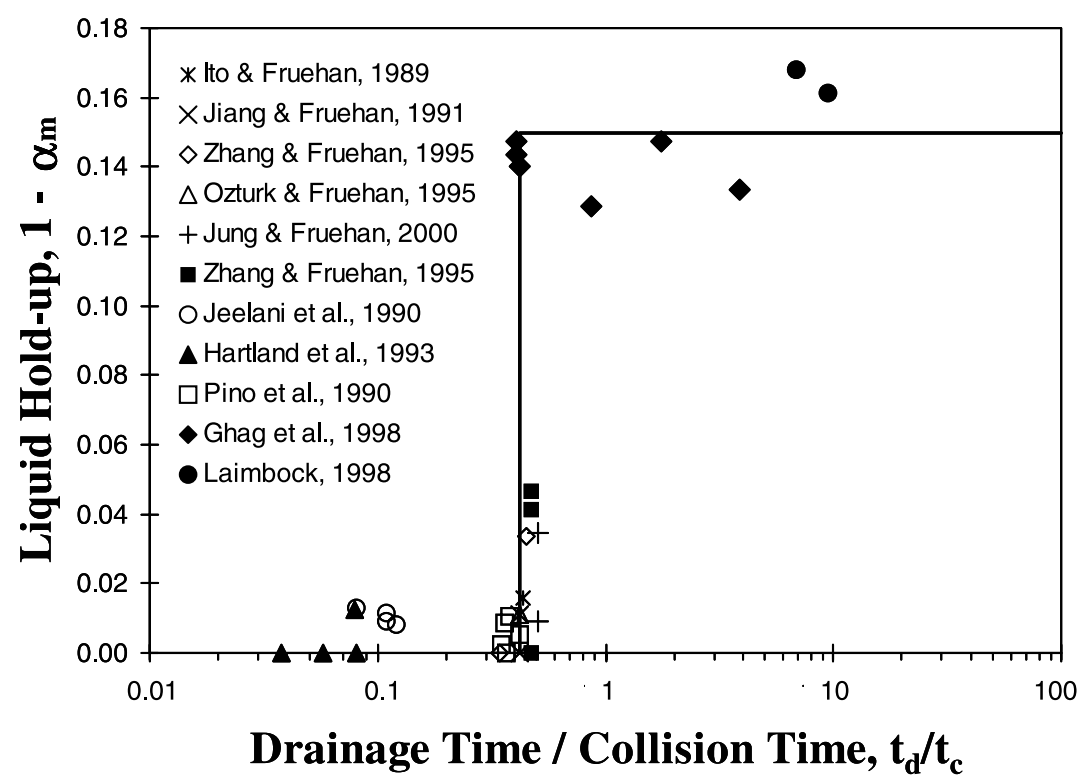

Fig. 4. Liquid hold-up for onset of foaming $\left(1-\alpha_{m}\right)$ as a function of the characteristic time ratio $t_{d} / t_{c}$.

experimental data of $j_{m}$ based on Eq. (24). Table 2 summarizes the physico-chemical properties and flow regimes for data collected from the literature. As one can see, experimental data cover a wide range of fluids, physico-chemical properties, bubble sizes and shapes, flow regimes, and hydrodynamic conditions.

\subsection{Regimes for onset of foaming}

Fig. 4 shows the plot of the minimum liquid hold-up for onset of foaming $\left(=\left[1-\alpha_{m}\right]\right)$ as a function of the ratio of the drainage time to the collision time $t_{d} / t_{c}$. Similarly,
Fig. 5 shows the variation of the minimum liquid hold-up as a function of the probability of coalescence of a rising bubble with a bubble at rest at the free surface as given by Eqs. (7), (9) and (10). In both figures, one can see a sharp transition in the liquid hold-up for onset of foaming corresponding to a characteristic time ratio of 0.42 and a probability of coalescence of $66 \%$. For the inertia dominated drainage, the transition expressed in terms of Weber number based on the terminal velocity occurs for $W e_{\infty}=5.6$. This value should be compared with $W e_{\infty}=3.3$ found by Duineveld for total inhibition of bubble coalescence in pure water [19], bearing in mind that inhibition of coalescence occurs

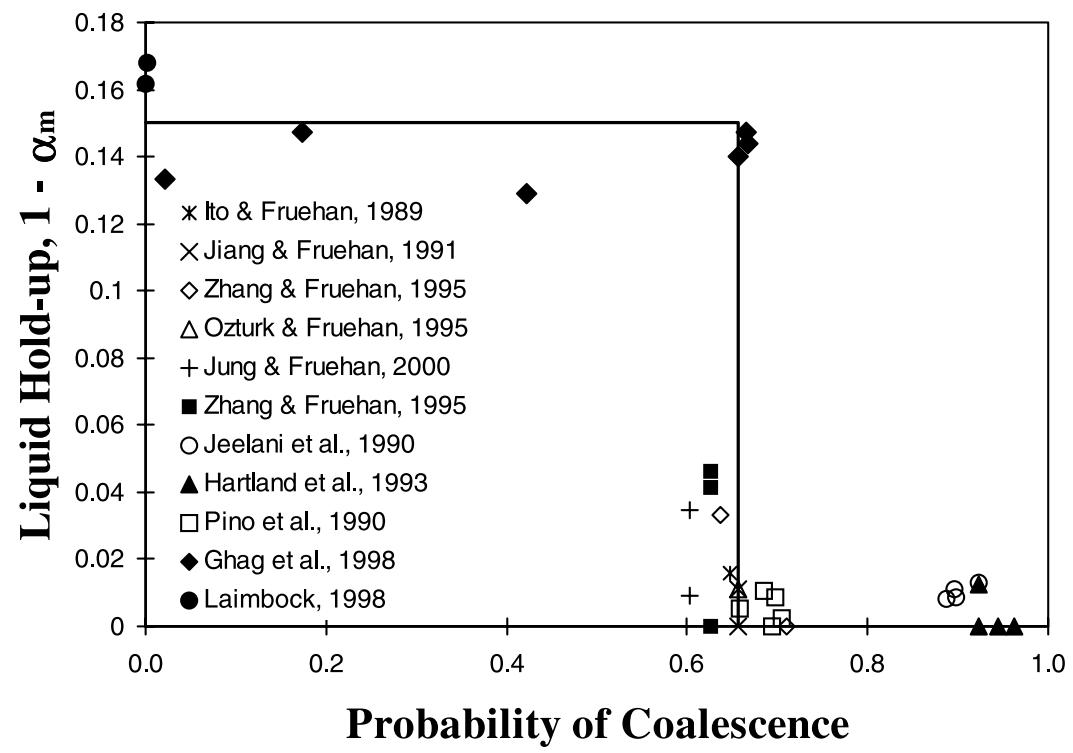

Fig. 5. Liquid hold-up for onset of foaming $\left(1-\alpha_{m}\right)$ as a function of the probability of coalescence between rising bubbles and bubbles at rest at the liquid free surface. 
Table 2

Summary of experimental data for minimum superficial velocity for onset of foaming reported in the literature

\begin{tabular}{|c|c|c|c|c|c|c|c|c|}
\hline Solution & Gas & $\sigma(\mathrm{mN} / \mathrm{m})$ & $\mu_{f}(\mathrm{mPa} \mathrm{s})$ & $\rho\left(\mathrm{kg} / \mathrm{m}^{3}\right)$ & $r_{0}(\mathrm{~mm})$ & Two-phase flow & $t_{d} / t_{c}$ range & References \\
\hline $40 \% \mathrm{CaO}-40 \% \mathrm{SiO}_{2}-5 \% \mathrm{FeO}-15 \% \mathrm{Al}_{2} \mathrm{O}_{3}$ & Argon & 463.0 & 398 & 2743 & $7.8-13.5$ & Distorted bubble & $\approx 0.47$ & Zhang and Fruehan [16] \\
\hline $48 \% \mathrm{CaO}-32 \% \mathrm{SiO}_{2}-10 \% \mathrm{FeO}-10 \% \mathrm{Al}_{2} \mathrm{O}_{3}$ & Argon & 477.2 & 381 & 2733 & 12 & Distorted bubble & 0.42 & Ozturk and Fruehan [1] \\
\hline $75 \mathrm{SiO}_{2}-15 \mathrm{NaO}_{2}-10 \mathrm{CaO}$ (wt.\%) glass & Air & $297.7-307.7$ & $7450-12100$ & $2346.6-2358.6$ & $15-20$ & Viscous (Stokes) & $6.9-12.4$ & Laimbock $[6]$ \\
\hline Water $+78-95 \%$ glycerinate + SDBS & $\mathrm{N}_{2}$ & $69.5-72.3$ & $46.5-520.8$ & $1204-1251$ & $0.7-1.1$ & Viscous (Stokes) & $0.42-3.9$ & Ghag et al. [9] \\
\hline $30 \% \mathrm{FeO}-42 \% \mathrm{SiO}_{2}-28 \% \mathrm{CaO}$ & Argon & 477.9 & 1605 & 3055 & 12 & Distorted bubble & 0.43 & Ito and Fruehan [13] \\
\hline $3 \% \mathrm{FeO}\left(\mathrm{CaO} / \mathrm{SiO}_{2}=1.25\right)$ & Argon & 477.2 & 381 & 2733 & 12 & Distorted bubble & 0.42 & Jiang and Fruehan [15] \\
\hline $0 \% \mathrm{FeO}\left(\mathrm{CaO} / \mathrm{SiO}_{2}=1.25\right)$ & Argon & 472.8 & 396 & 2693 & 12 & Distorted bubble & 0.42 & Jiang and Fruehan [15] \\
\hline $30 \% \mathrm{CaO}-60 \% \mathrm{SiO}_{2}-10 \% \mathrm{CaF}_{2}$ & Argon & 338 & $533[31]$ & 2534 & 13 & Distorted bubble & 0.47 & Zhang and Fruehan $[17,32]$ \\
\hline $34.78 \% \mathrm{CaO}-33.76 \% \mathrm{SiO}_{2}-22.52 \% \mathrm{FeO}-8.94 \% \mathrm{MgO}$ & Argon & 502 & 270 & 2958 & 17 & Distorted bubble & 0.51 & Jung and Fruehan $[18]$ \\
\hline $37.39 \% \mathrm{CaO}-35.57 \% \mathrm{SiO}_{2}-20.87 \% \mathrm{FeO}-6.17 \% \mathrm{MgO}$ & Argon & 493 & 291 & 2936 & 17 & Distorted bubble & 0.51 & Jung and Fruehan [18] \\
\hline Water $+10 \%$ glycerinate Marlophen 89 and 812 & $\mathrm{~N}_{2}$ & $32.0-41.1$ & 1.22 & 1014 & $0.5-0.78$ & Viscous (wake) & $0.08-0.12$ & Jeelani et al. [7] \\
\hline Water + sucrose AR + glycerol SLR + aerosol OT & $\mathrm{N}_{2}$ & 26 & 20 & 1220 & 3.9 & Distorted bubble & 0.41 & Hartland and Barber [33] \\
\hline Water $+10 \%$ glycerinate Marlophen 89 and 812 & $\mathrm{~N}_{2}, \mathrm{NO}_{x} \mathrm{Xe}, \mathrm{CO}_{2}$ & $32.0-41.1$ & 1.22 & 1014 & $0.5-0.78$ & Viscous (wake) & $0.04-0.08$ & Hartland et al. [8] \\
\hline $\begin{array}{l}\text { Tap water, aqueous glycerine, kerosene, kerosene } \\
+ \text { surfactants }\end{array}$ & Air & $20.4-72.2$ & $0.89-3.42$ & $808-1104$ & $3.4-5.7$ & Viscous (wake) & $0.35-0.42$ & Pino et al. [11] \\
\hline
\end{tabular}


over a range of Weber numbers and depends on the liquid properties.

Moreover, it is interesting to note that the void fraction for onset of foaming $\alpha_{m}$ obtained for large characteristic time ratio $t_{d} / t_{c}$ (i.e. for small probability of bubble coalescence) is about 0.85 . This is consistent with the fact that the onset of foaming corresponds to a physical situation between an interface free of foam and a layer of foam with a void fraction of 0.74 at the liquid/foam interface as schematically represented in Fig. 3. In the former case, the void fraction above the liquid/surrounding interface is unity while in the latter case it corresponds to the maximum packing of spheres of same size. Experimental data indicate that, in the regime of low probability of bubble coalescence, bubbles are spherical and viscous forces dominate the thinning of the film separating bubbles.

Since the parameter $n$ is empirically determined, a sensitivity study has been performed with an estimated $\pm 20 \%$ error in the value of $n$ given in Table 1 . The results show similar trend as that previously discussed with a regime transition occurring at the same characteristic time ratio of 0.42 . For large characteristic time ratio $t_{d} / t_{c}$, the average void fraction for onset of foaming $\alpha_{m}$ varies between 0.78 and 0.92 while that for large characteristic time ratio is larger than 0.99 .

We speculate that coalescence of rising bubbles with a bubble at rest at the liquid free surface is a key phenomenon in the onset of foaming. If rising bubbles coalesce instantaneously with the bubble(s) at rest at the free surface, the bubble resulting from single or multiple coalescences becomes too large to be stable and bursts. Only a few large bubbles are present at the liquid free surface at a given time and foam cannot form. If bubble coalescence is somehow inhibited then bubbles can accumulate at the liquid free surface and a foam layer starts forming.

For small characteristic time ratio $\left(t_{d} / t_{c}<0.42\right)$ and large probability of coalescence $(P>66 \%)$ bubbles tend to coalesce more leading to larger bubbles that are forced to change from spherical to polyhedral shape in order to be stable [28]. Foams consisting of polyhedral bubbles can assume void fraction for onset of foaming $\alpha_{m}$ close to unity. In the static foam layer surfactants stabilize the liquid lamellae separating the bubbles thus reducing the probability of coalescence of bubbles and enabling the foam to be stable at large void fractions. In contrast, if bubble coalescence is strongly inhibited because of slow drainage and/or Plateau suction effects, bubbles keep their spherical shape and the maximum void fraction should correspond to the maximum packing of identical spheres (i.e. 0.74). The drift-flux model predicts that foam should form for any arbitrarily small superficial gas velocity and for small superficial gas velocities the foam should have a void fraction close to unity (see Fig. 1). The disagreement with experimental observations described earlier can be explained as follows: (1) for large bubble coalescence probability (i.e. small $t_{d} / t_{c}$ ), the functioning point predicted by the drift-flux model is unstable due to large unstable bubbles present and quickly bursting at the free surface and (2) for small bubble coalescence probability (i.e. large $t_{d} / t_{c}$ ) a functioning point in the foamy regime cannot be reached by the system since bubbles remain spherical leading to a foam morphology that cannot assume void fractions close to unity.

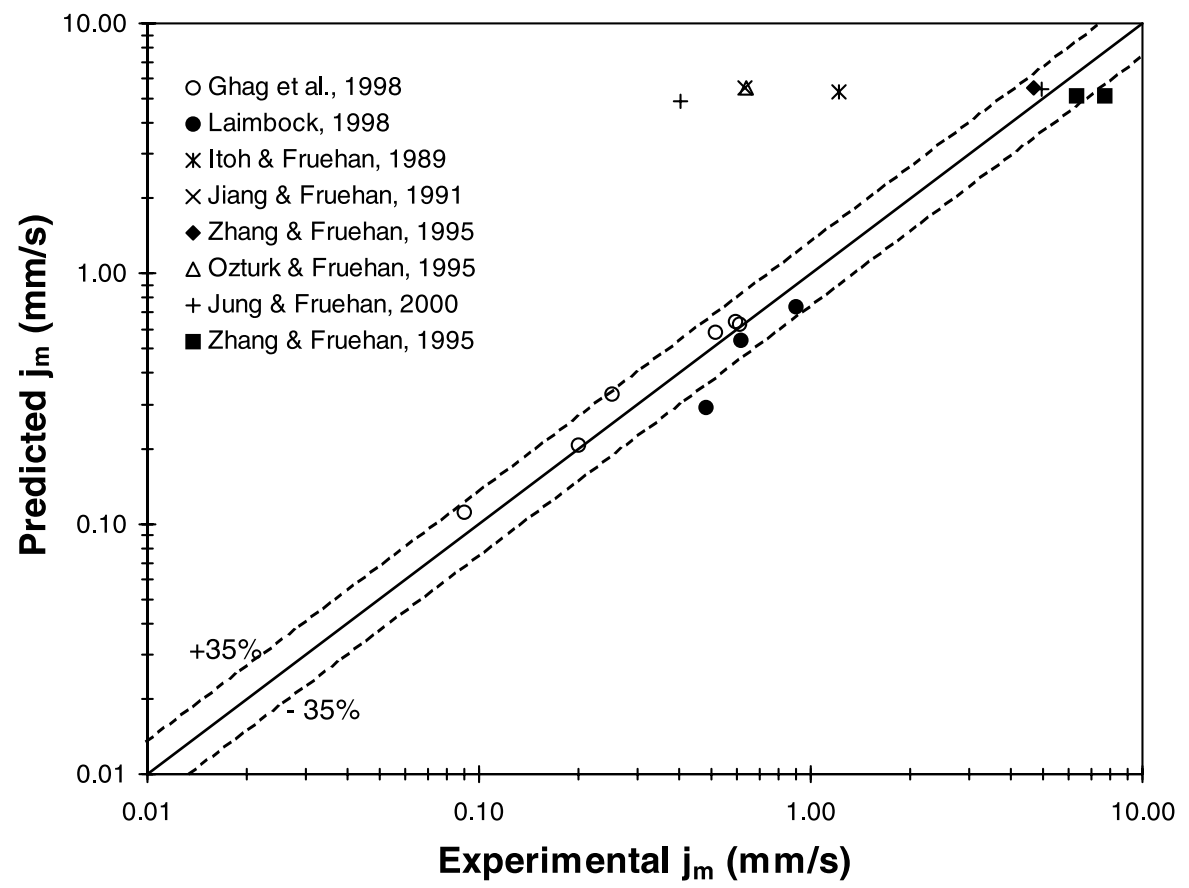

Fig. 6. Comparison between experimental and predicted minimum superficial gas velocity for onset of foaming for viscosity dominated drainage, i.e. small characteristic time ratio $t_{d} / t_{c}$. 
Experimental observations for the inertia dominated drainage showed that an increase in the viscosity of the liquid phase increases the coalescence rate owing to a reduction in the bubble velocity and to the formation of larger stable bubbles as reviewed by Pino et al. [11]. Finally, Bukur et al. [10] observed that no foam was generated when the liquid viscosity is too large. They attributed this phenomena to the fact that 'coalescence increases with the liquid viscosity'. Thus, a stable foam layer does not form due to the absence of a large number of small bubbles that coalesced before reaching the interface. These speculations are confirmed by the present analysis. Indeed, for inertia dominated drainage, an increase in the viscosity reduces the bubble velocity and thus the Weber number. Then, according to Eqs. (7) and (9), the probability of coalescence of a rising bubble with a bubble at rest at the free surface is close to unity.

Fig. 6 compares the experimental data for the minimum superficial gas velocity for onset of foaming $j_{m}$ with the model predictions given by Eq. (24), using a maximum void fraction $\alpha_{m}$ of 0.85 in the case of small probability of coalescence $(P<66 \%)$ between a rising bubble and a bubble at rest at the liquid free surface. Good agreement between experimental data and the model predictions is found. When the probability of coalescence is larger, the model predictions for $j_{m}$ appear to be very sensitive to the void fraction $\alpha_{m}$ for onset of foaming.

The analysis developed in the present work does not use explicitly the initial liquid height; however, we speculate that this height has an influence on the superficial gas velocity for the onset of foaming $j_{m}$. Indeed, if the liquid depth is large enough, bubbles have time to reach their terminal velocity, and the onset of foaming should not depend significantly on the initial liquid height. In contrast, if the initial liquid depth is small, the velocity at which bubbles reach the interface will depend on the initial liquid depth. However, determining the approach velocity of a bubble in shallow baths requires the complex analysis of the bubble rise in which transient forces such as the Basset force and the added mass force must be accounted for [5,29]. Unfortunately, the complete formulation of the transient forces acting on a rising bubble is not available at the present time [29]. Moreover, the effect of the liquid height seems to be negligible for the experimental data collected in the literature and used in the present work. Thus, as a first order approximation, the approaching bubble velocity can be assumed to equal the terminal velocity $v_{\infty}$.

The present study does not consider the effect of the container size on the onset of foaming since the model assumes uniform velocity and void fraction profiles at any given cross-section perpendicular to the two-phase flow. Such an assumption may not be valid for large containers where uniform void fraction and velocity are more difficult to obtain experimentally and where spreading of the foam over the liquid bath may be significant.

Finally, Guitian and Joseph [24] showed that the injection of the liquid phase at the bottom of the column (i.e. co-current flow with $j_{f}>0$ ) delays the foam formation to larger superficial gas velocities and the condition for onset of foaming can be written as:

$j_{g}=a+b j_{f}$

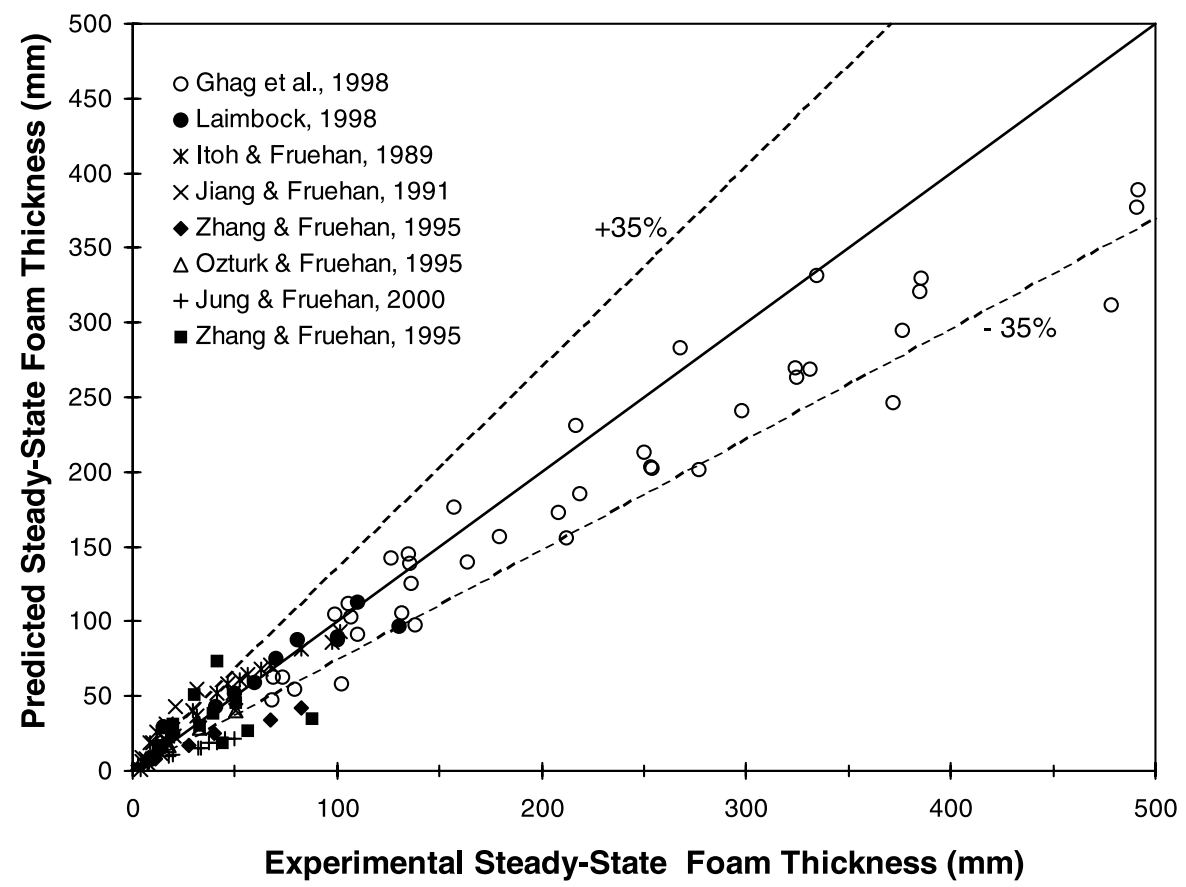

Fig. 7. Comparison between experimental and predicted steady-state thickness for viscosity dominated drainage, i.e. small characteristic time ratio $t_{d} / t_{c}$. 
where $a$ and $b$ are constants independent of the superficial velocities $j_{g}$ and $j_{f}$. The present work focuses on the constant $a=j_{m}$ and considered quiescent liquid for which $j_{f}=0$.

\subsection{Steady-state foam thickness}

Pilon et al. [3] developed a semi-empirical correlation for predicting the steady-state foam thickness of viscous fluids ( $\mu \geq 46 \mathrm{mPa}$ ) based on the dimensional analysis of the drainage equation [30]. They found that the steady-state thickness $H_{\infty}$ is proportional to $\left(j_{g}-j_{m}\right)^{0.8}$ and expressed as [3]:

$H_{\infty}=2905 \frac{\sigma}{r^{2.6}} \frac{\left[\mu_{f}\left(j_{g}-j_{m}\right)\right]^{0.8}}{\left(\rho_{f} g\right)^{1.8}}$

where $H_{\infty}$ is the steady-state foam thickness, $r_{0}$ is the average bubble radius at the bottom of the foam layer, $\sigma$ is the surface tension of the liquid-gas system, $j$ is the superficial gas velocity, $\rho$ is the density of the liquid phase, and $g$ is the specific gravity. However, $j_{m}$ was determined from experimental data such as those shown in Fig. 2 which limits the prediction capability of Eq. (27). Fig. 7 compares the experimental data for the steady-state foam thickness with the predictions of Eq. (27) combined with the present model for the superficial gas velocity $j_{m}$ for experimental data having characteristic time ratio $t_{d} / t_{c} \geq 0.42$. The superficial gas velocity for onset of foaming was determined from Eq. (24) using a maximum void fraction for onset of foaming $\alpha_{m}$ equals 0.85 . Given the complexity of the physical phenomena taking in liquid-gas foams, Fig. 7 indicates that the model gives reasonable and similar predictions for the steady-state foam thickness as those obtained when $j_{m}$ was determined experimentally. Note that (1) the smaller the probability of bubble coalescence, i.e. the larger the characteristic time ratio $t_{d} / t_{c}$, the better the model's predictions, and (2) the largest deviations from the experimental data occur for small gas influxes, i.e. for small steady-state foam thicknesses. The model's predictions for the steady-state foam thickness falls within $\pm 35 \%$ error bound for characteristic time ratio $t_{d} / t_{c}>0.5$. Therefore, the present work completes the formulation of a self-contained model for the steady-state thickness of pneumatic foams generated from viscous fluids over a wide range of physico-chemical properties and operating conditions (see Table 2).

\section{Concluding remarks}

A model is presented for predicting the superficial gas velocity for onset of foaming, and the analysis has provided a better understanding of the physical mechanisms responsible for foam formation. The analysis is based on the one-dimensional drift-flux model for gravity driven flow with no wall shear. Inhibition of coalescence between rising bubbles and bubbles at rest at the free interface has been identified as a key mechanism for explaining the onset of foaming. Expression for the probability of coalesce can be found in the literature [20]. The analysis is based on experimental data collected from the literature which covers a wide range of physico-chemical properties, bubble sizes and shapes, and flow regimes and the following conclusions can be drawn:

- Two different regimes for the onset of foaming have been identified: (1) for low probability of coalescence, i.e. large characteristic time ratio $t_{d} / t_{c}$, bubble coalescence is strongly inhibited, and the foam formation occurs for relatively large void fractions of about 0.85 , and (2) for large probability of coalescence, i.e. small characteristic time ratio $t_{d} / t_{c}$, bubbles tend to coalesce more leading to their distortion and the onset of foaming for void fractions close to unity.

- A semi-empirical correlation for the superficial gas velocity for onset of foaming $j_{m}$ at low probability of coalescence between rising bubbles and bubbles at rest at the free surface $(P<0.66)$ is given by Eq. $(24)$. The associated maximum void fraction for onset of foaming $\alpha_{m}$ is equal to 0.85 . Good agreement between the model predictions and experimental data is observed for both the superficial gas velocity for onset of foaming $j_{m}$ and the steady-state foam thickness when $j_{g}>j_{m}$.

\section{Acknowledgements}

This work was supported by the US Department of Energy/Glass Industry/Argonne National Laboratory/University collaborative research project.

\section{Appendix A. Nomenclature}

$\begin{array}{ll}a, b & \text { constants, Eq. (26) } \\ A & \text { container cross-sectional area } \\ C_{D} & \text { drag coefficient } \\ f\left(r^{*}\right) & \text { functions of the dimensionless radius, Eq. (23) } \\ g & \text { specific gravity } \\ H_{\infty} & \text { steady-state foam thickness } \\ j & \text { superficial velocity } \\ j_{g f} & \text { drift-flux } \\ K & \text { constant Eq. (5) } \\ n & \text { constant determined experimentally Eq. (5) } \\ N_{\mu} & \text { viscosity number Eq. (11) } \\ P & \text { Probability of coalescence } \\ Q & \text { volumetric flux } \\ R e & \text { Reynolds number Eq. (12) } \\ r & \text { bubble radius } \\ r^{*} & \text { dimensionless bubble radius Eq. (11) } \\ t_{c} & \text { bubble collision duration time } \\ t_{d} & \text { characteristic time for drainage } \\ v & \text { velocity } \\ V & \text { relative velocity of centers of colliding bubbles }\end{array}$


$V_{g j} \quad$ drift velocity

We Weber number Eq. (8)

$\begin{array}{ll}\text { Greek } & \text { symbols } \\ \alpha & \text { void fraction } \\ \sigma & \text { surface tension } \\ \mu & \text { dynamic viscosity } \\ \psi\left(r^{*}\right) & \begin{array}{l}\text { function of the dimensionless bubble radius } r^{*} \\ \text { Eq. (16) } \\ \rho\end{array} \\ \text { density }\end{array}$

\section{Subscripts}

$g \quad$ refers to the gas phase

$f \quad$ refers to the liquid phase

$m \quad$ refers to the minimum conditions for onset of foaming

$t \quad$ refers to the transition between spherical and distorted bubbles

$\infty \quad$ refers to conditions of a single bubble rising in an infinite medium

\section{References}

[1] B. Ozturk, R.J. Fruehan, Effect of temperature on slag foaming, Metallurgical and Materials Transactions B 26B (1995) 1086-1088.

[2] J.J. Bikerman, Foams, Springer, New York, 1973.

[3] L. Pilon, A.G. Fedorov, R. Viskanta, Steady-state foam thickness of liquid-gas foams, Journal of Colloid and Interface Science 242 (2001) 425-436.

[4] G.B. Wallis, One-Dimensional Two-Phase Flow, McGraw-Hill, New York, 1969.

[5] M. Ishii, One-dimensional drift-flux model and constitutive equations for relative motion between phases in various two-phase flow regimes, Argonne National Laboratory Report ANL-77-47, 1977.

[6] P.R. Laimbock, Foaming of glass melts, Ph.D. thesis, Technical University of Eindhoven, Eindhoven, The Netherlands, 1998.

[7] S.A.K. Jeelani, S. Ramaswami, S. Hartland, Effect of binary coalescence on steady-state height of semi-batch foams, Transaction of the Institution of Chemical Engineers-Part A 68 (1990) 271-277.

[8] S. Hartland, J.R. Bourne, S. Ramaswami, A study of disproportionate effects in semi-batch foams-II. Comparison between experiment and theory, Chemical Engineering Science 48 (1993) 1723-1733.

[9] S.S. Ghag, P.C. Hayes, H.-G. Lee, Physical model studies on slag foaming, ISIJ International 38 (1998) 1201-1207.

[10] D.B. Buruk, D. Petrovic, J.G. Daly, Flow regime transitions in a bubble column with a paraffin wax as the liquid medium, Industrial and Engineering Chemistry Research 26 (1987) 1087-1092.

[11] L.Z. Pino, M.M. Yepez, A.E. Saez, G. De Drago, An experimental study of gas holdup in two-phase bubble columns with foaming liquids, Chemical Engineering Communications 89 (1990) 155-175.

[12] Y.T. Shah, S. Joseph, D.N. Smith, J.A. Ruether, On the behavior of the gas phase in a bubble column with ethanol-water mixtures, Industrial Engineering and Chemistry Process Design and Development 24 (1985) 1140-1148.
[13] K. Ito, R.J. Fruehan, Study of the foaming of $\mathrm{CaO}-\mathrm{SiO}_{2}-\mathrm{FeO}$ slags: Part I. Foaming parameters and experimental results, Metallurgical and Materials Transactions B 20B (1989) 509-514.

[14] K. Ito, R.J. Fruehan, Study of the foaming of $\mathrm{CaO}-\mathrm{SiO}_{2}-\mathrm{FeO}$ slags: part II. Dimensional analysis and foaming in iron and steelmaking processes, Metallurgical and Materials Transactions B 20B (1989) $515-521$.

[15] R. Jiang, R.J. Fruehan, Slag foaming in bath smelting, Metallurgical and Materials Transactions B 22B (1991) 481-489.

[16] Y. Zhang, R.J. Fruehan, Effect of bubble size and chemical reactions on slag foaming, Metallurgical and Materials Transactions B 26B (1995) 803-812.

[17] Y. Zhang, R.J. Fruehan, Effect of gas type and pressure on slag foaming, Metallurgical and Materials Transactions B 26B (1995) 1089-1091.

[18] S.-M. Jung, R.J. Fruehan, Foaming characteristics of BOF slags, ISIJ International 40 (2000) 348-354.

[19] P.O. Duineveld, Bouncing and coalescence of bubble pairs rising at high Reynolds number in pure water or aqueous surfactant solutions, Applied Scientific Research 58 (1998) 409-439.

[20] A.K. Chesters, The modelling of coalescence processes in fluidliquid dispersions: a review of current understanding, Transactions of the Institution of Chemical Engineers-Part A 69 (1991) 259270.

[21] R.D. Kirkpatrick, M.J. Lockett, The influence of approach velocity on bubble coalescence, chemical Engineering Science 29 (1974) 2363-2373.

[22] J.W. Kim, W.K. Lee, Coalescence behavior of two bubbles in stagnant liquids, Journal of Chemical Engineering of Japan 20 (1987) 448453.

[23] M. Ishii, N. Zuber, Drag force and relative velocity in bubbly, droplet or particulate flows, The American Institute of Chemical Engineers Journal 25 (5) (1979) 843-855.

[24] J. Guitian, D. Joseph, How bubbly mixture foam and foam control using fluidized bed, International Journal of Multiphase Flow 24 (1) (1998) 1-16.

[25] M. Ishii, T.C. Chawla, Local drag laws in dispersed two-phase flow, Argonne National Laboratory Report ANL-79-105, NUREG/CR1230, 1979.

[26] Y. Ogawa, D. Huin, H. Gaye, N. Tokumitsu, Physical model of slag foaming, ISIJ International 33 (1) (1993) 224-232.

[27] R. Clift, J.R. Grace M.E. Weber, Bubbles, Drops, and Particles, Academic Press, New York, 1978.

[28] A.J. De Vries, Morphology, coalescence, and size distribution of foam bubbles, in: R. Lemlich (Ed.), Adsorptive Bubble Separation Techniques, vol. 1, Academic Press, New York, NY, 1972, pp. 7-31.

[29] J.J.M. Magnaudet, The forces acting on bubbles and rigid particles, in: ASME Fluids Engineering Division Summer Meeting, FEDSM'97, Part 16, Vancouver, Canada, 22-26 June 1997, vol. 16, pp. $1-7$.

[30] A. Bhakta, E. Ruckenstein, Decay of standing foams: drainage, coalescence and collapse, Advances in Colloid and Interface Science 70 (1997) 1-124.

[31] K. Wu, S. Chu, W. Qian, Q. Niu, Investigation into rheological characteristic and foaming behaviour of molten slags, Steel Research 70 (7) (1999) 248-251.

[32] Y. Zhang, R.J. Fruehan, Effect of carbonaceous particles on slag foaming, Metallurgical and Materials Transactions B 26B (1995) 813-819.

[33] S. Hartland, A.D. Barber, A model for cellular foam, Transactions of the Institution of Chemical Engineers 52 (1974) 43-52. 